\title{
VÍNCULOS TRANSFRONTERIZOS: VIDA, MOVILIDAD Y COMERCIO EN EL BARRIO BOLIVIANO DE IQUIQUE, CHILE
}

\author{
Crossborder Linkages: Life, Mobility, and Trade \\ in Iquique's Bolivian Neighborhood
}

\author{
Marcela Tapia Ladino ${ }^{1}$ \\ Fernanda Chacón Onetto ${ }^{2}$
}

\begin{abstract}
Resumen. El estudio del Barrio Boliviano de Iquique permite captar los vínculos laborales e interacciones económicas que dan lugar a prácticas sociales fronterizas y que se expresan en movilidades en el territorio que no siempre implican el establecimiento o sedentarización en el lugar de destino. De modo que el barrio se convierte en un dispositivo comercial fronterizo y en parte de un territorio circulatorio donde las brechas de desarrollo, las diferencias salariales y el efecto llamada del mercado de trabajo explican la circulación de personas por motivos comerciales y laborales más allá de los litigios diplomáticos entre Bolivia y Chile.
\end{abstract}

Palabra claves: frontera, circulación, prácticas sociales fronterizas, territorio circulatorio.

\begin{abstract}
The study of Iquique's Bolivian neighborhood allows one to identify labor linkages and economic interactions that give rise to social border practices and are expressed in mobilities in the territory that do not always imply establishment or settlement in a place within the place of destination. The neighborhood thus becomes a border trade device and part of a circulatory territory in which development gaps, salary differences, and the labor market call effect explain the circulation of people for commercial and labor reasons that go beyond the diplomatic litigation between Bolivia and Chile.
\end{abstract}

Keywords: border, circulation, social border practices, circulatory territory

1 Profesora Titular y Directora del Instituto de Estudios Internacionales INTE de la Universidad Arturo Prat. Iquique, Chile.

2 Socióloga por la Universidad Arturo Prat. Iquique, Chile. 
El presente trabajo ${ }^{3}$ tiene por objetivo analizar las prácticas sociales transfronterizas que tienen lugar en un barrio particular de lquique, el barrio boliviano. El objetivo de este trabajo es mirar cómo se constituye un espacio transfronterizo en la ciudad debido a la circulación de comerciantes y migrantes que llegan al barrio y que gracias a sus prácticas producen procesos de vinculación que conectan al norte de Chile con Bolivia. Existe un importante desarrollo sobre los procesos de interacción y complementariedad en espacios contiguos debido las diferencias producidas por las brechas de desarrollo y el gap económico que supone cruzar la frontera ${ }^{4}$. Asimismo desde la lectura de la geografía social y del transnacionalismo migrante se ha atendido no sólo a las interacciones que unen a las personas a través de las fronteras, sino también a las redes y recorridos que éstos describen y a los tipos de movilidades que establecen sin que ello suponga necesariamente sedentarización en el lugar de destino ${ }^{5}$. Por tanto el análisis que proponemos incluye no sólo a la movilidad laboral que se ha venido estudiando en Chile de manera prolífica, sino también a la movilidad comercial "muy adecuada a la evolución general de los intercambios y a la globalización de las economías"

El debate actual de los estudios de frontera advierte sobre cómo las prácticas sociales que despliegan las personas que cruzan y circulan por el linde vinculan a territorios adyacentes a pesar del carácter separador de la frontera en clave geopolítica. Especialmente en un contexto de litigios vigentes con Bolivia que reaviva el nacionalismo en ambos países y nos retrotrae a fórmulas decimonónicas de la resolución de litigios. Por tanto, revisar los procesos de interacción "por abajo" o desde la perspectiva de los habitantes invita a pensar la frontera, la movilidad y los espacios afectados por la internacionalidad las regiones fronterizas- como construcciones sociales en permanente transformación, es decir, como un proceso "abierto y contingente"

El barrio en estudio se ubica en el centro histórico de la ciudad y comprende la calle Esmeralda a la altura del 1000, entre las calles Amunátegui y Arturo Fernández y la intersección de calle Juan Martínez. La particularidad de este barrio iquiqueño es que en él habitan, transitan y circulan extranjeros de origen sudamericano, en especial bolivianos, aunque no exclusivamente. El barrio, denominado por los habitantes de la zona central de la ciudad como boliviano, surgió con el impulso

3 Este trabajo es resultado del Proyecto FONDECYT № 1130642 titulado "Migrantes latinoamericanos en Chile: precariedad e informalidad en enclaves étnicos, agricultura y construcción" cuya Investigadora Responsable es la Dra. Carolina Stefoni de la Universidad Alberto Hurtado.

4 ALEGRÍA, Tito. La ciudad y los procesos transfronterizos entre México y Estados Unidos; IDEM. Juntos pero no revueltos: ciudades en la frontera México-Estados Unidos.

5 HEYMAN, Josiah. Construcción y uso de tipologías: movilidad geográfica en la frontera MéxicoEstados Unidos; TARRIUS, Alain. Migrantes pobres y globalización de las economías: el transnacionalismo migratorio en Europa meridional.

6 Ibidem, p. 102.

7 BENEDETTI, Alejandro. Lugares de frontera y movilidades comerciales en el sur sudamericano. Una aproximación multiescalar, p. 38. 
de la Zona Franca de Iquique ZOFRI en 1975 y de expansión y crecimiento en los años 80. La ZOFRI, en articulación con el puerto de lquique, abrió la región al comercio internacional y ha actuado de puente para el comercio fronterizo y transfronterizo. A partir de este vínculo económico y comercial la región y el norte de Chile comercian con Bolivia, Paraguay y Brasil principalmente.

Desde el 2000 Bolivia ha repuntado en indicadores económicos y el aumento de los precios de las materias primas se ha traducido en un auge para el país entre los principales factores explicativos ${ }^{8}$. En este contexto general es que el barrio boliviano acoge y atiende de manera espontánea a comerciantes bolivianos que cruzan la frontera para comprar mercancías en la ZOFRI para luego vender en las distintas ciudades del país dando lugar en los últimos años, a lo que Tassi denomina como "desborde de la economía popular boliviana" ${ }^{\text {. }}$. Este comercio transfronterizo explica en parte el surgimiento de una élite o grupo aymara y mestizo, unos de larga trayectoria comercial ${ }^{10}$, otros comerciantes emergentes y algunos casos de importadores que compran directamente desde China vía Iquique y Arica ${ }^{11}$. Estos comerciantes han desarrollado una institucionalidad propia relativamente sólida con una marcada profundidad histórica anclada al territorio transfronterizo.

Como hemos señalado en otras publicaciones, referirnos al norte de Chile implica considerar la configuración de sus fronteras y los constantes litigios diplomáticos como consecuencia de lo mismo, lo que nos retrotrae con frecuencia a definiciones decimonónicas sobre los límites ${ }^{12}$. Por tanto, se trata de un espacio en constante tensión que discurre entre la interacción social y la integración económica por un lado, especialmente por la movilidad de sus habitantes ${ }^{13}$ y el comercio fronterizo; y las demandas y procesos de fronterización o refuerzo de las fronteras, por otro.

A este análisis debemos añadir el aumento de la migración sudamericana hacia Chile y la aceleración experimentada en años recientes, hecho que ha motivado una preocupación por el fenómeno migratorio en las Ciencias Sociales y de las autoridades de gobierno ${ }^{14}$. Sin embargo, y como hemos

8 TASSI, Nico et alii. El desborde económico popular en Bolivia. Comerciantes aymaras en el mundo global.

9 Ibidem.

${ }^{10}$ LLANQUE, Ricardo, VILLCA, Edgar. Qamiris aymaras. Desplazamiento e inclusión de elites andinas en la ciudad de Oruro.

11 TASSI, Nico et alii. "Hacer plata sin plata". El desborde de los comerciantes populares en Bolivia.

${ }^{12}$ OVANDO, Cristian. La noción de soberanía en las propuestas bolivianas para la salida al mar: el caso de Arica trinacional.

13 TAPIA, Marcela. Frontera, movilidad y circulación reciente de peruanos y bolivianos en el norte de Chile Estudios atacameños.

${ }^{14}$ Según la actualización de cifras demográficas del Instituto Nacional de Estadísticas INE, la migración hacia Chile creció un 160\% entre el año 2002 y 2012 (Matus, 21 de septiembre de 2014). En este mismo sentido las estimaciones del Departamento de Extranjería y Migraciones 
señalado en otro lugar, la presencia de extranjeros fronterizos ha sido un rasgo distintivo de las regiones nortinas explicada por la incorporación de los territorios mencionados y por la atracción que ha ejercido la economía regional en el espacio circunvecino ${ }^{15}$.

A partir de la discusión reciente sobre frontera y región fronteriza por una parte y sobre migración y movilidad por otro, este artículo analiza el lugar que ocupan las prácticas sociales fronterizas en la región de Tarapacá que despliegan los comerciantes, migrantes, habitantes y actores conexos en su actividad en el barrio boliviano. El afán es observar fenómenos fronterizos que quedan fuera de la noción de migración y que tensionan el concepto de frontera para atender a los procesos de circulación y vinculación en torno a la frontera. Asimismo interesa revisar la expresión que adquiere esta interacción en el barrio boliviano de la ciudad de lquique para ponerlo en relación con la discusión sobre barrio migrante o enclave étnico, entre otras nociones. Se trata de mirar un aspecto menos explorado de los vínculos fronterizos como son el comercio y las conexiones transfronterizas expresadas en movilidad de población en la región y la ciudad, pero que tienen un correlato transfronterizo y transnacional. Este afán, como se mencionó anteriormente, se conecta con un fenómeno reciente como es el surgimiento y desbordamiento de la economía popular altiplánica que ha dado origen a una rica clase social aymara cuyos vínculos comerciales se conectan con la región y con el mundo a través de la Zona Franca de Iquique ${ }^{16}$.

En este sentido, si bien el análisis privilegia el nivel regional y el despliegue territorial de dichas prácticas, se tendrá en cuenta una mirada multinivel o multiescalar del fenómeno puesto que la actividad comercial se relaciona con procesos de internacionalización y de integración de los mercados y su concreción a partir del funcionamiento de un barrio particular de la ciudad. En este sentido se reconoce que los vínculos comerciales poseen una expresión global ineludible, sin embargo en este caso el foco se ubica en una de las regiones emplazadas en el límite entre Chile y Bolivia -Tarapacá- y en su capital, Iquique, donde existe un marcado sentido de frontera debido a la demanda marítima boliviana.

El marco temporal de análisis considera las primeras décadas del siglo XXI hasta hoy, sin perder vista el contexto histórico de más largo plazo. La metodología de investigación corresponde a etnografía en el barrio boliviano, realización de entrevistas semi-estructuradas y revisión y análisis de prensa

DEM del Ministerio del Interior, señalan que para el año 2014 los extranjeros en Chile ascendían a 477.450 personas, muy lejos de los 184.464 del año 2002, lo que representa un 2,7\% de la población total (<http://www.extranjeria.gob.cl/>).

15 TAPIA, Marcela. Migración y movilidad de los trabajadores fronterizos en Tarapacá durante el ciclo del nitrato. 1880-1930; IDEM. Extranjeros fronterizos en las regiones extremas de Chile: entre migración y circulación. 1990-2014.

${ }^{16}$ TASSI et alii, El desborde económico..., op. cit.; TASSI et alii, “Hacer plata sin plata..., op. cit. 
local, en este caso de La Estrella de Iquique desde 1990 al $2007^{17}$. Asimismo se utilizaron fuentes secundarias como memorias y anuarios económicos además de datos estadísticos sobre economía regional y nacional.

El artículo se organiza de la siguiente forma, la primera parte corresponde a la discusión teórica sobre circulación, frontera y barrios o enclaves étnicos como marco para analizar lo que ocurre en el barrio boliviano. La segunda parte se refiere al análisis del barrio como un lugar de llegada de comerciantes y migrantes -en el sentido más tradicional de la acepción-y de migrantes móviles, que van y vienen y su paso o estancia en dicho espacio. Luego el análisis se detiene en el barrio como plataforma de servicios para los comerciantes bolivianos que llegan a Iquique a comprar a la ZOFRI y pasan por el barrio, comen, cambian dinero o pernoctan para luego volver a Bolivia. Por último, el análisis se detiene en el barrio como lugar de residencia de migrantes, condiciones de vida y oportunidades laborales.

\section{Frontera y prácticas sociales en un ¿barrio migrante? ¿enclave étnico? o ¿territorio circulatorio?}

Como marco teórico para el análisis nos remitimos a la discusión más reciente sobre circulación, frontera y barrios o enclaves étnicos que nos parecen que son pertinentes para el estudio del Barrio Boliviano, deteniéndonos en los puntos de convergencia de dichos cuerpos teóricos. La complejidad del fenómeno a estudiar invita a proponer un marco unificado de estudio que vincula circularidad, frontera y barrio y las prácticas sociales y económicas que las relacionan. Como señala Portes nuestro desafío es aplicar varias nociones de mediana escala que son propuestos como un juego de herramientas para analizar el fenómeno escogido ${ }^{18}$.

Respecto del debate sobre las fronteras ésta tiene un amplio desarrollo y se caracteriza por ser multi o transdisciplinario, por tanto posee distintas perspectivas, énfasis y miradas que de alguna manera no tienen límites precisos ${ }^{19}$. En los últimos años el debate sobre las fronteras ha transitado desde la preocupación por la línea, como separador de pueblos que incluye y excluye al mismo tiempo; a la preocupación por los procesos de interacción, intercambio y encuentro ${ }^{20}$. Para este trabajo adscribimos al desarrollo más reciente sobre los estudios fronterizos o border studies, el cual centra su atención en las perspectivas de quienes habitan, cruzan o viven en la frontera ${ }^{21}$. Esta mirada desde el territorio no significa renunciar a la consideración de lo nacional, sino por el contrario implica reconocer el

\footnotetext{
17 El año 2007 la Región de Tarapacá fue dividida para crear la XV Región de Arica y Parinacota.

${ }_{18}$ PORTES, Alejandro. La Sociología en el hemisferio. Hacia una nueva agenda conceptual.

19 TAPIA, Marcela, GONZÁLEZ, Adriana. Regiones fronterizas, migración y los desafíos para los Estados nacionales latinoamericanos.

${ }^{20}$ NEWMAN, David. On borders and power: A theoretical framework; ZAPATA-BARRERO, Ricard, FERRER-GALLARDO, Xavier. Las fronteras en la época de la movilidad.

${ }^{21}$ GIELIS, Ruben. Borders make the difference: Migrant transnacionalism as a border experience.
} 
cruce entre lo local, nacional y global como una superposición de capas que se entrelazan y dan lugar a lo binacional, lo transfronterizo y lo transnacional.

En este caso interesa la movilidad fronteriza y las prácticas comerciales que tienen o han tenido como referente la frontera y más propiamente la región fronteriza. En este sentido la frontera es al menos "una realidad dual" 22 y para su análisis es preciso "reconocer la tensión originada entre la idea de la indivisibilidad de la soberanía estatal y la colindancia y continuidad de una trama social que no se acaba dentro de los bordes de la nación, debido justamente a una serie de antecedentes históricos y culturales de la existencia de las naciones"23. Por tanto nos preocupa la trama social que tejen quienes se mueven o cruzan para quedarse, para ir y volver o para circular. De este modo las prácticas en torno a la frontera configuran un dominio regional "que se refiere a los intercambios sociales, a su vez económicos, culturales y demográficos, en niveles tanto infra como interestatales" ${ }^{24}$. Estas prácticas pueden tener distintos fines, entre ellas, las prácticas productivas que engloban la búsqueda de empleo, comercio (formal e informal) y las distintas estrategias para la obtención de ingresos y aumentar el rendimiento de los recursos o el abaratamiento de los costos de la vida. Como señala Benedetti existe una relación dialéctica entre frontera y movilidad, en tanto "los lugares de frontera atraen movilidades y las movilidades dan vida a esas localizaciones" 25 . En especial cuando las brechas de desarrollo son notorias "el espacio fronterizo, como yuxtaposición de territorialidades con asimetrías estructurales y coyunturales, se transforma en recurso y su cruce en una estrategia de reproducción"26. Así, a pesar del carácter separador de la frontera y la desigualdad que produce, la colindancia invita al cruce y favorece los movimientos de población. Como afirma el autor que seguimos:

Las movilidades aquende y allende la frontera son relaciones sociales que producen y reproducen esas continuidades. La movilidad espacial (en adelante sólo movilidad) es un tipo particular de relación social ligada al cambio de localización de las personas y/o de los bienes; es una estrategia de reproducción social a partir de la relación entre lugares, definiendo trayectorias espaciotemporales complejas ${ }^{27}$.

La noción de movilidad, como hemos señalado es más amplia que el concepto de migración en tanto la primera es un tipo de movimiento inserto en un campo más amplio de desplazamientos ${ }^{28}$. La migración nos remite casi siempre

${ }^{22}$ MORALES, Abelardo. Desentrañando fronteras y sus movimientos transnacionales entre pequeños estados. Una aproximación desde la frontera Nicaragua-Costa Rica, p. 185.

${ }^{23}$ Ibidem, p. 186.

24 Ibidem.

${ }^{25}$ BENEDETTI, op. cit., p. 37.

${ }^{26}$ Ibidem, p. 38.

27 Ibidem, p. 36.

${ }^{28}$ TAPIA, Marcela, PARELLA, Sonia. Las regiones fronterizas para el estudio de la migración y la circulación. Un análisis de dos casos ilustrativos. 
al establecimiento en el lugar de destino, temporal o definitivo, lo que a menudo implica un cambio de residencia, en cambio la movilidad no implica un cambio de residencia porque se realiza por períodos cortos, días o menos de una semana, en cualquier caso las personas retornan constantemente a su lugar de origen como es el caso de los commuters en la frontera de EE.UU y México. Así "la combinación del espacio de movimiento, el tiempo de estancia y el motivo de la movilidad definen y diferencian un movimiento (tipo de movilidad) de una migración" ${ }^{29}$. En los espacios fronterizos es frecuente este tipo de movilidades con distintos fines, cruces de la frontera para comprar más barato, atención médica, ocio, estudios o trabajo ${ }^{30}$. Los factores que impulsan el cruce periódico son "individuales y familiares y los factores macro estructurales, como son las regulaciones migratorias y los mercados laborales que demandan población trabajadora" ${ }^{\prime 1}$.

Sin embargo, no es sólo migración y movilidad lo que encontramos en las regiones fronterizas, sino también circulación migratoria, noción proveniente de la geografía social francesa que pone en entredicho la distinción entre aquí y allá y otorga al movimiento espacial un sentido social. Como señala Tarrius esta noción "implica la socialización de espacios de soporte de las prácticas de movilidad"32, lo que permite captar movimientos más fluidos y variados que no suponen necesariamente una ruptura entre dos espacios sino el surgimiento de un territorio circulatorio. De modo que "las identificaciones y referencias territoriales ya no se ubican "aquí y alli", sino en el mismo territorio constituido por las circulaciones" ${ }^{\prime 33}$.

\section{El barrio como lugar de llegada y circulación de migrantes y comerciantes}

Es posible analizar la presencia urbana de extranjeros a partir de la noción del espacio como un lugar construido socialmente. Dicha construcción se produce a partir de la constante interacción entre los valores, normas, símbolos, significados y prácticas que se objetivan materialmente en el territorio ${ }^{34}$. De esta forma, los lugares utilizados por los migrantes y/o comerciantes fronterizos para residir definitiva o temporalmente, trabajar, consumir o llevar a cabo transacciones económicas, sociabilizar, etc., son espacios y territorios socialmente creados, que "expresan las estrategias de los actores migrantes frente a un espectro muy

${ }^{29}$ CASTILLO, Manuel, NÁJERA, Jéssica. México como país de origen, tránsito y destino de migrantes, una revisión a partir de la EMIF NORTE y la EMIF SUR, p. 20.

30 PARELLA, Sonia. Una exploración de las prácticas transfronterizas en la zona urbana Caléxico (Estados Unidos)-Mexicali (México).

${ }^{31}$ CASTILLO, NÁJERA, op. cit., p. 20.

32 TARRIUS, Alain. Pobres en migración, globalización de las economías y debilitamiento de los modelos integradores: el transnacionalismo migratorio en Europa meridional, p. 139.

33 TAPIA, PARELLA, op. cit., p. 179.

34 DI MEO, Guy. Géographie tranquille du quotidien. Une analyse de la contribution des sciences ociales et de la géographie à l'étude des pratiques spatiales. 
variable de oportunidades y presiones" ${ }^{\prime 35}$. La relación de los migrantes con los espacios urbanos y la comunidad han sido teorizados a partir de la noción de barrios migrantes y barrios étnicos provenientes de la geografía, de los enclaves étnicos y de la economía étnica y economía migrante, desde la antropología. Ambos corpus tienen elementos que resultan útiles para comprender el lugar que ocupan los barrios en la movilidad y la circulación de personas.

La noción de barrio fue acuñada en EE.UU y definida de manera genérica como "un área, sector o distrito de la ciudad con características históricas y culturales, esto es, como espacio de identidad social"36. Esta idea incluye desde la tipificación física del espacio, es decir un área claramente delimitada, hasta una comunidad barrial donde se desarrolla un sentido de pertenencia y colectividad que se diferencia de otros barrios. Esto último se "acentúa por patrones de segregación urbana por las fuerzas etnoculturales" ${ }^{\prime \prime 2}$. Estos casos se tratan de migración asentada de larga data consolidada en el país de destino. La modalidad de barrio étnico es un tipo de barrio migrante con una clara vocación de comunidad y formado sobre la base "de las relaciones personales, familiares o sociales consolidadas por las cadenas y redes migratorias"38.

La noción de enclave étnico fue acuñada para Alejandro Portes en sus estudios sobre empresas cubanas en Miami para aludir a

Grupos de inmigrantes que se concentran en una localización espacial específica y ponen en marcha toda una variedad de empresas para abastecer a su propio mercado étnico y/o a la población general. Su característica básica es que una proporción significativa de la fuerza de trabajo inmigrante trabaja en empresas dirigidas por otros inmigrantes ${ }^{39}$.

Este tipo de negocios se concentraban en el espacio atrayendo a proveedores y clientes produciendo un efecto multiplicador de dicha economía. Además se constituyó en un nicho laboral donde los recién llegados aprendieron un oficio que les permitió aspirar a conseguir su negocio propio más tarde constituyéndose en un mecanismo de movilidad social. Recientemente Portes revisitó este aporte teórico a los estudios migratorios y concluyó que en el caso de los cubanos en Miami la economía de enclave fue un instrumento para hacerse un espacio social y económicamente respetable en la sociedad norteamericana especialmente para la primera generación. Sin embargo, no fue así para la segunda generación quienes se vieron excluidos de las ventajas y oportunidades provenientes de

\footnotetext{
${ }^{35}$ BABY-COLLIN, Virginia et alii. Mujer, movilidad y territorialización. Análisis cruzado de las migraciones internacionales en México y Bolivia, p. 153.

${ }^{36}$ SASSONE, Susana, MERA, Carolina. Barrios de migrantes en Buenos Aires: Identidad, cultura y cohesión socioterritorial, p. 1.

37 Ibidem, p. 2.

38 Ibidem.

${ }^{39}$ BUCKLEY, Monica. "Amos del universo" y "aves de paso" en la conformación del centro urbano, p. 1048.
} 
las redes del enclave ${ }^{40}$. Así el enclave étnico fue concebido como una zona de concentración geográfica de empresas cuyos propietarios, fuerza de trabajo y clientes mayoritariamente migrantes o pertenecientes a un grupo étnico específico. Los fundamentos teóricos en torno al barrio étnico reconocen en él no sólo un espacio sino también un aspecto relacional y comunitario que emerge a partir de un proceso migratorio de largo aliento ${ }^{41}$.

Por su parte el concepto de economía étnica fue desarrollado por Light ${ }^{42}$, Bonacich y Modell ${ }^{43}$ para designar a todas las pequeñas empresas cuyos propietarios y empleados pertenecen a un mismo grupo étnico ${ }^{44}$. A diferencia del concepto de enclave, en las economías étnicas no es requisito la concentración geográfica de las empresas, sino la utilización de redes de apoyo, vínculos basados en la confianza, ciertos tipos de ayuda familiar, etc,de forma estratégica y rentable para el desarrollo de su actividad económica ${ }^{45}$. Este último punto converge con las nociones teóricas sobre el enclave étnico en tanto las redes urdidas en él para el caso de la primera oleada de migrantes cubanos en Miami se constituyeron en una plataforma de movilidad laboral ascendente $^{46}$. El concepto de economía migrante es aún más amplio que las nociones descritas anteriormente en tanto no es condición necesaria que los empleadores y la fuerza de trabajo formen parte del mismo grupo étnico, esto ya el objetivo de dicho constructo reside en "valorar las interacciones de la empresa étnica con el espacio urbano" ${ }^{\prime 7}$.

\subsection{El Barrio Boliviano de Iquique}

El barrio boliviano está ubicado en la zona histórica de la ciudad de Iquique (en el antiguo barrio Matadero), en Esmeralda 1000 entre las calles Amunategui y Juan Martínez. Desde los años 90 comenzó un proceso de deterioro en términos de infraestructura debido a la pérdida de sus antiguos habitantes y la salida de sus residentes a barrios nuevos ubicados en la zona sur de la ciudad ${ }^{48}$. De esta manera el centro fue ocupado gradualmente por familias de menores ingresos -motivados principalmente por la disminución

\footnotetext{
${ }_{40}$ PORTES, Alejandro, SHAFER, Steven. Revisitando la hipótesis del enclave: Miami veinticinco años después.

${ }^{41}$ SASSONE, MERA, op. cit.

${ }^{42}$ LIGHT, Ivan. Ethnic Enternrise in America.

${ }^{43} \mathrm{BONACICH}$, Edna, MODELL, John. The Economic Basis of Ethic Solidarity in the Japanese American Community.

${ }^{44}$ BUCKLEY, op. cit.

${ }^{45}$ GARCÉS, Alejandro. Migración peruana en Santiago: prácticas, espacios y economías.

${ }^{46}$ PORTES, Alejandro, JENSEN, Leif. The Enclave and the Entrants: Patterns of Ethnic Enterprise in Miami Before and After Mariel.

47 BUCKLEY, op. cit., p. 106.

${ }^{48}$ LABBÉ, Gricel. Del gueto al hipergueto en el centro y pericentro de la ciudad de Iquique.
} 
del costo de las viviendas- y por actividades informales evidenciadas en la ocupación del espacio público por parte de ferias y vendedores ambulantes.

Plano área central de lquique y Barrio Boliviano ${ }^{49}$
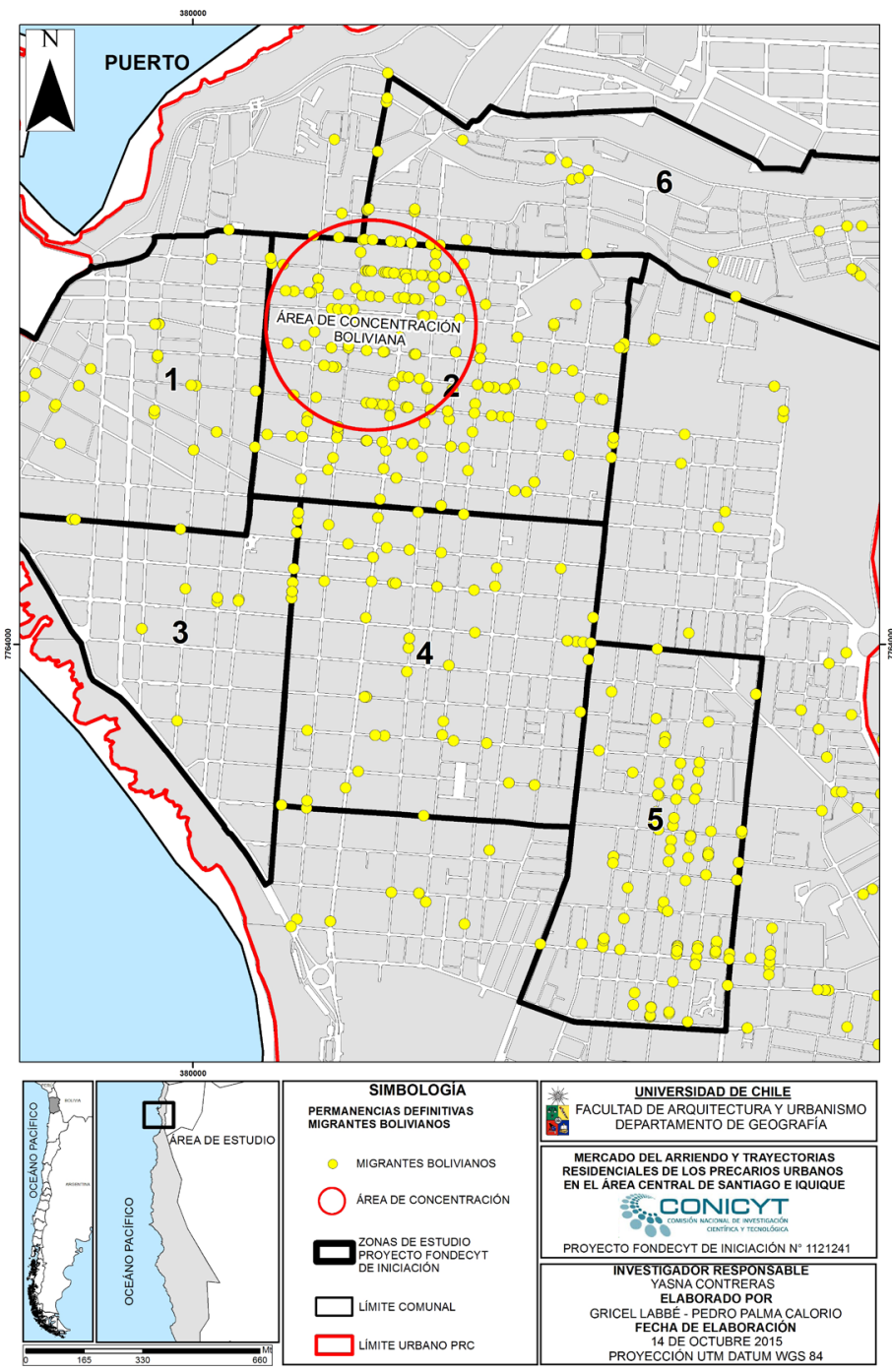

Una de las características del barrio, y en general de la zona centro de lquique, es la percepción de inseguridad, incertidumbre y su estigmatización como un espacio de tráfico de drogas y delincuencia donde prima la ilegalidad,

${ }^{49}$ Agradecemos la colaboración de la Dra. Yasna Contreras y su equipo de investigación FONDECYT №112141 por la elaboración de este plano. 
aspectos destacados por la prensa local. Con el aumento de las migraciones sudamericanas el barrio se fue constituyendo en espacio de acogida de los recién llegados o de aquellos que llegaban a la ZOFRI a comprar, especialmente de origen boliviano. En la medida que mejoró la conectividad con Bolivia a través del paso fronterizo de Colchane a fines del siglo XX aumentó el número de buses y pasajeros que llegaban a Iquique. Los primeros buses llegaron a la calle Esmeralda a la altura del 1000 y con los años este espacio se consolidó como el paradero de las "flotas", como les denominan los bolivianos ${ }^{50}$. Poco a poco se fueron instalando negocios para atender a los pasajeros, servicios de taxis, residenciales, restaurantes, centrales telefónicas y casas de cambio, entre otras, hasta alcanzar su actual fisonomía.

El principal paso fronterizo por el que ingresan bolivianos a Tarapacá es el de Colchane ubicado a $262 \mathrm{kms}$ de Iquique en la Cordillera de los Andes a una altura de 3695 msnm. El año 2000 ese paso registró un total de 61.808 entradas y salidas, para aumentar el 2010 a 302.566 entradas y salidas. El año recién pasado el total de cruces por ese paso fue de 574.623, lo que significa que un aumento $1000 \%$ en 14 años y ubicándose entre los pasos más transitados de Chile ${ }^{51}$.

De este modo el Barrio Boliviano se fue convirtiendo en un espacio de paso y tránsito de migrantes y comerciantes preferentemente bolivianos, aunque no exclusivamente, a un espacio de establecimiento temporal en algunos casos y definitivo en otros. Así, se ha ido constituyendo por distintos flujos económicos, culturales, de personas, entre otros, en tanto los bolivianos y sudamericanos han hecho de este territorio un espacio desde el cual llevan a cabo diversas actividades económicas, las que principalmente están asociadas al comercio y a la prestación de servicios (formal e informal).

En el barrio existen aproximadamente 40 locales de prestación de servicios, de los cuales sólo 3 pertenecen a chilenos, mientras que el resto de los dueños son de nacionalidad boliviana. La mayoría de ellos no reside en la ciudad, sin embargo viajan constantemente a supervisar sus negocios. Lo característico es que estos locales son atendidos en su mayoría por bolivianos. Del total de los negocios señalados, aproximadamente 18 empresas son de transporte cuyos principales destinos son Cochabamba, Santa Cruz, Oruro y La Paz. Según entrevistas de campo la mayoría de estas empresas se encuentran representadas ante la ley bajo una misma persona, quien trabaja también en el Barrio.

Existen además alrededor de 12 locales que funcionan al mismo tiempo como centro de llamados e internet y/o casas de cambio, de los cuales 3 de ellos

${ }^{50}$ PEREIRA, A., URIBE, A. Migración comercial boliviana. Acercamiento exploratorio y descriptivo sobre el fenómeno de la migración comercial boliviana y su vinculación con la Zona Franca de Iquique, en la comuna de Iquique.

51 Información obtenida de la Policía de Investigaciones remitida el 29 de octubre de 2015 por la Ley $N^{\circ} 20.285$ sobre derecho de acceso a la Información. 
pertenecen a chilenos. Algunas residenciales también funcionan como casas de cambio, lo que dificulta la estimación. Es posible encontrar también alrededor de 5 restaurantes étnicos en el sector, los cuales preparan principalmente platos típicos bolivianos a bajos precios. El resto de los negocios se dividen entre alojamientos, un local que funciona como garaje y un bar.

El tipo de comercio y de actividades económicas que ofrece el barrio indican que su existencia obedece principalmente a la satisfacción de las necesidades de los propios migrantes y comerciantes transfronterizos. En este sentido se ajusta en parte a la noción de barrio migrante porque no se trata de una migración consolidada que busca establecerse en el lugar, sino por el contrario que busca los servicios para continuar sus trayectorias o para ir y venir nuevamente, pero que tiene un sentido de pertenencia. "[Mis clientes] son los que viajan... ellos están por decirte un día o dos días y se van ellos y llegan otros pasajeros" (M, Vendedora de frutas, 40 años).

Ahora bien, la ubicación del barrio en una zona central de la ciudad tiene la ventaja de la proximidad para quienes lo habitan o permanecen en él más tiempo, es decir, es un lugar donde se puede "articular trabajo, residencia y redes sociales" 52 . Sin embargo, es al mismo tiempo una zona residencial precaria y tugurizada donde el arriendo y el subarriendo proliferan, principalmente entre migrantes sudamericanos a lo que se suman los problemas de delincuencia y tráfico de drogas. Como señaló una de las entrevistadas, "Los robos, asaltos son los problemas [del barrio], que no dejan trabajar la gente a veces llega de tan lejos a comprar y los dejan sin nada" (M, Vendedora de frutas, 40 años).

La prensa local también consigna esta situación e informa de los constantes asaltos a los que se ven expuestos los comerciantes bolivianos y los problemas que deben enfrentar por el escaso equipamiento y seguridad del barrio:

Más de 500 bolivianos viajan diariamente a Iquique para efectuar compras en la Zona Franca y llevar mercaderías a su país. Sin embargo su estada en el puerto se ha transformado en algo muy desagradable por la serie de asaltos que sufren.

Son las principales víctimas de delincuentes debido a que portan gran cantidad de dinero y los antisociales saben que ellos no presentan las denuncias por falta de tiempo.

Además deben vivir una gran odisea todos los días, pues tienen que esperar los buses a la intemperie en el sector comprendido entre las calles Juan Martínez, desde San Martín hasta Esmeralda.

Todos los días a partir de las 20 horas en adelante comienzan a reunirse para obtener sus pasajes y alistar las maletas con la finalidad de iniciar el viaje a Oruro. La travesía dura 17 horas y pasan por Colchane a las 5 de la mañana, donde deben bajar y esperar un par de horas para la respectiva revisión.

${ }_{52}$ LABBÉ, op. cit., p. 25. 
Al ser consultados, manifestaron su deseo que en Iquique exista un terminal y mejor seguridad de lo contrario ya están pensando en optar por viajar a comprar a Ilo, Perú (La Estrella de Iquique, 26 de abril de 1998, Estamos aburridos de los asaltos)

El barrio es al mismo tiempo fuente de trabajo para la población boliviana que llega a Iquique y quiere trabajar, especialmente en al ámbito de la hostelería, restaurantes y negocios varios. Quienes trabajan en los hospedajes regularmente alojan en el lugar de trabajo, comen en restaurantes del sector a precios bajos. Esto permite al trabajador no sólo ahorrar parte importante de su sueldo, sino también muchas veces le permite al empleador extender la jornada de trabajo de sus empleados, situación experimentada también por las mujeres que trabajan en el servicio doméstico. El tipo de actividades desarrolladas no requiere una fuerza de trabajo cualificada, sino más bien mayor disponibilidad para realizar largas jornadas laborales, flexibilidad por parte del trabajador y mano de obra barata. Esta tríada hace del migrante o del recién llegado que quiere probar suerte mayores expectativas salariales, un trabajador ideal para insertarse en este tipo de trabajos ${ }^{53}$.

Para las mujeres y hombres bolivianos que se instalan en lquique a trabajar, pero que no residen en el barrio, se vinculan a él por los viajes de retorno o visitas, especialmente por quienes migran solos. Algunas de las entrevistadas señalaron que viajan periódicamente a sus ciudades o localidades de origen con el propósito de reencontrarse con la familia o hacer frontera, es decir activar la visa de turista cuando está por vencer ${ }^{54}$. Quienes han reagrupado a sus familias en Iquique son las que realizan menor cantidad de viajes, por ejemplo una vez al año, mientras que las que poseen familia en Bolivia o trabajan por tiempos cortos lo hacen regularmente, por ejemplo cada mes, cada tres meses y en algunos casos cuando distancia de viaje es mayor, cada seis meses dependiendo el caso.

\section{El barrio como plataforma de servicios para comerciantes}

Como señalamos al barrio no sólo llegan los migrantes como tal, es decir que tienen por objetivo buscar un trabajo y permanecer en Iquique de manera temporal o definitiva, sino también comerciantes fronterizos cuya estadía en la ciudad es temporal. Normalmente la estancia está condicionada a la duración de la actividad económica que realizan, que va desde los dos días hasta una semana aproximadamente. Se trata de comerciantes que provienen de distintas ciudades de Bolivia y que vienen a comprar a la ZOFRI una variedad de artículos entre los que destacan ropa, electrodomésticos y autos. Mientras

\footnotetext{
${ }^{53}$ SOLIMANO, Andrés, TOKMAN, Víctor. Migraciones internacionales en un contexto de crecimiento económico: el caso de Chile.

54 TAPIA, Marcela, RAMOS, Romina. Mujeres migrantes fronterizas en Tarapacá a principios del siglo XXI. El cruce de las fronteras y las redes de apoyo.
} 
permanecen en la ciudad buscan abaratar los costos de alojamiento y comida y las mejores alternativas las encuentran en el Barrio. La alta circulación de personas ha beneficiado y potenciado las actividades económicas informales como la venta de frutas o la instalación de una feria nocturna que abastece de los últimos detalles para el viaje como a ropa y mercadería de todo tipo. Como señaló una de las entrevistadas: "Vienen [al barrio] a comprar ciertos días... Yo creo que les conviene trabajar aquí pues..." (M, Vendedora de fruta, 40 años).

Al mismo tiempo el Barrio se constituye en una red de apoyo para quienes habitan o circulan, principalmente para los compatriotas de origen boliviano o los vecinos peruanos y los propios chilenos. Algunas de las informantes mantienen contactos y relaciones con bolivianos provenientes de su localidad de origen en la ciudad, los que también han elegido al barrio como sector de residencia, mientras otras han ido formando lazos importantes de apoyo y solidaridad entre compatriotas, los que también residen o circulan en el sector. Este tipo de lazos y redes ha permitido a las informantes hacer más llevadera la vida en el barrio como señaló otra informante: "La única ayuda que he recibido es la de la Julia porque cuando yo llegué a Iquique de Vallenar no tenía plata me tendió la mano" (C., Mucama, 30 años).

Todas las entrevistados en el estudio llegaron al país con escasos recursos económicos (entre 100 y 400 USD), y si bien algunas han llegado acompañadas de cercanos (familiares o amigos), lo limitado de sus redes en Iquique las hace más proclive a vivir situaciones de vulnerabilidad, lo que ha sido atenuado gracias a los lazos de apoyo que han ido construyendo durante su estadía en la ciudad, especialmente en el barrio ${ }^{55}$. Entre las distintas formas de apoyo destacan: alojamiento gratuito, préstamos para subsistir y/o la posibilidad de insertar al recién llegado al mercado laboral gracias a las redes establecidas por los migrantes que llevan más tiempo. Otras formas son más sutiles, y pueden facilitar, por ejemplo, el trabajo de los nuevos migrantes: donación de agua para el puesto de una de las informantes por parte de una vecina del sector, la posibilidad de reservar alimentos en otros locales comerciales ${ }^{56}$, entre otros. Para una de las informantes las redes que ha ido formando en el lugar la han ayudado a subsistir sin tener trabajo con jornada completa: "Sí, a veces yo le ayudo a lavar los platos o a pelar las papas en cualquier cosa les ayudo y me dan [de comer]" (E., Vendedora de frutas, 35 años).

De esta forma el barrio boliviano entendido como territorio circulatorio, actúa a su vez como una plataforma de inserción laboral para muchos migrantes móviles, es decir, los que van y vienen y para comerciantes transfronterizos que satisfacen las necesidades propias del viaje de negocios. Los contactos interpersonales

\footnotetext{
${ }_{55}$ RIESCO, Alberto. Enclaves y economías étnicos desde la perspectiva de las relaciones salariales.

${ }^{56}$ Una de las mujeres vende frutas con yogurt en la calle. Las medidas sanitarias obligan a que el yogurt conserve cierta temperatura, por lo que cuando aparece carabineros por el sector a fiscalizar, la señora reserva el yogurt en los locales próximos a su puesto para así evitar el control.
} 
establecidos durante el cruce de la frontera, el capital social construido en la circulación o saber circular y las confianzas establecidas por la convivencia en el barrio son centrales para comprender los modos de incorporación social, económica o laboral en la ciudad. En el caso de los migrantes predomina la precariedad laboral y residencial especialmente por la inserción en la economía informal y el acceso al mercado de la vivienda del subarriendo. Asimismo la rotación en distintos trabajos y el uso de la visa de turista para permanecer impiden la inserción laboral formal y favorecen la circulación constante, aspectos que se condicen con el proyecto migratorio de la mayoría de las entrevistadas. En el caso de los comerciantes, los viajes de compras no implican un apego al lugar porque esa movilidad es netamente comercial y el afán es abaratar los costos del viaje y aumentar el rendimiento de la compra de mercancías.

\section{El barrio, de lugar de paso a lugar de residencia}

\subsection{Trayectoria laboral}

Como advierten los Informes de organismos internacionales y la literatura gris sobre migración y mercado de trabajo, los migrantes internacionales, especialmente recién llegados, tienden a incorporarse en la economía informal o sumergida en nichos laborales específicos, regularmente de baja aceptabilidad para los nacionales ${ }^{57}$. Un rasgo común en los flujos migratorios internacionales es la consideración de los extranjeros como una mano de obra barata y flexible en el marco de una creciente segmentación del mercado de trabajo y la demanda por cuidados y servicios de proximidad ${ }^{58}$. En la migración y la movilidad fronteriza ocurre de manera similar si tenemos en cuenta que Chile se constituye en un destino migratorio por el atractivo que ejerce el desempeño económico del país ${ }^{59}$. De esta forma, las trayectorias laborales de quienes cruzan la frontera para buscar trabajo están fuertemente vinculadas a las oportunidades laborales existentes y las condiciones de vida se relacionan con las posibilidades a las que pueden aspirar con los recursos obtenidos. En este sentido, se aprecian dinámicas laborales descendentes y ascendentes entre las informantes en general, aunque se observan mejoras sustanciales en las condiciones laborales y de vida en directa relación con el tiempo de residencia en Chile y regularización de la estadía.

Las trayectorias laborales de las entrevistadas muestran un modo de incorporación al mercado laboral marcado especialmente por el género. Las informantes se han insertado principalmente en el sector terciario de la economía, como empleadas domésticas o mucamas en residenciales en la

\footnotetext{
57 SOLÉ, Carlota, PARELLA, Sonia. La inserción de los inmigrantes en el mercado de trabajo. El caso español.

${ }^{58}$ PARELLA, Sonia. El trasvase de desigualdades de clase y etnia entre mujeres: los servicios de proximidad; IDEM. Segregación laboral y 'vulnerabilidad social' de las mujeres inmigrantes a partir de la interacción entre clase social, género y etnia.

59 SOLIMANO, TOKMAN, op. cit.
} 
modalidad de internas o puertas adentro. Se insertan también como garzonas en restaurantes del barrio bolivianos y en otros casos como independientes, por ejemplo como costureras o vendedoras ambulantes. Varias de las entrevistadas tenían experiencia migratoria previa, por ejemplo a Argentina, desempeñándose en trabajos similares a los descritos en Chile.

La incorporación laboral de las entrevistadas en el barrio se caracteriza por la precariedad; la mayoría de las informantes no posee contrato de trabajo, las jornadas laborales son extensas entre 10 a las 12 horas diarias y regularmente trabajan por el sueldo mínimo o menos. Aunque las condiciones laborales en Bolivia no son mejores que las que encuentran en Chile, las entrevistadas perciben mayores beneficios en Iquique debido al efecto que produce la brecha de desarrollo en los salarios. En este sentido, en Chile las entrevistadas pueden aspirar a ganar el doble o más de lo que ganarían por el mismo trabajo en Bolivia:

[Trabajando en una hostal] Podía ganar como 150.000 allá en Bolivia y acá gano 420.000" (C, Mucama, 30 años).

"Acá hay más trabajo y pagan más. [En Bolivia ganaba] 800 bolivianos, como 220 dólares" (E. Vendedora de fruta, 35 años).

"Mira cuando yo llegué estaba bien, no me quejo pero ahora no por el dólar, yo viajé y la plata de aquí es lo mismo de allá porque antes era mejor. Claro y acá gano 400 dólares pero claro la vida es más cara y las deudas... no es tan factible, o sea conviene en términos de sueldo pero no de vivir acá" (R., Asesora del hogar, 33 años).

Cabe destacar que la mayoría de las migrantes entrevistadas vive en Iquique hace poco más de un año. Quienes llevan más tiempo -cuatro años o más- han tenido la oportunidad de insertarse al mercado laboral con un contrato de trabajo y la regularización de sus papeles. Sin embargo, es recurrente el cambio en los trabajos, es decir, rotación laboral y por tanto inestabilidad. Esto se explica porque no todos los entrevistados tienen por objetivo establecerse por largo tiempo en Iquique sino satisfacer necesidades familiares precisas, lo que se traduce en proyectos migratorios de corta duración. Por tanto, lo que encontramos es fundamentalmente movilidad laboral acotada sin establecimiento permanente en la ciudad y con idas y venidas frecuentes.

La única persona entrevistada que posee un trabajo más permanente es una vendedora de frutas que tiene un puesto en la calle y que trabaja como independiente. Lleva 17 años en Chile y aunque señala que en la venta ambulante las ganancias no dan lo mismo que un trabajo como empleada domésticas puertas adentro, valora las ventajas de trabajar como independiente, especialmente por la posibilidad de controlar los propios tiempo, "Es que es mejor porque sola me mando, ya no ando mirando el reloj a veces cuando quiero salgo y cuando no quiero no salgo" (M., Vendedora de fruta, 47 años). 


\section{Conclusiones}

El estudio del barrio boliviano de lquique ha supuesto un desafío desde el punto de vista teórico puesto que ha obligado a poner en relación los aportes de las teorías migratorias y de los estudios de frontera para buscar los puntos de convergencia que permitan caracterizar y definir lo que ocurre en dicho espacio. Desde las teorías migratorias hemos señalado que la noción de migración es estrecha en tanto no da cuenta suficientemente de los fenómenos que ocurren en una región fronteriza donde la sedentarización no es el rasgo distintivo o necesariamente típico. Aunque la historia regional evidencia una configuración social multinacional de larga duración, especialmente fronteriza ${ }^{60}$, la historia reciente muestra más fluidez y ductilidad de los movimientos de población. Por tanto lo que encontramos en el estudio del barrio boliviano son movilidades fundamentalmente comerciales y laborales que se caracterizan por la circularidad en el contexto de la internacionalización de la economía y el efecto llamada de un mercado laboral fronterizo. La circularidad está dada por la oportunidad de compras de mercancías en la Zona Franca de Iquique, las brechas salariales producto de las asimetrías del espacio fronterizo, la contigüidad y la mayor conectividad para el cruce de la frontera.

Desde los estudios de frontera advertimos una mayor preocupación por las interacciones que ocurren a nivel territorial, sin embargo en el caso estudiado los litigios y las demandas están presentes a nivel nacional recordando la estricta integridad del territorio y la función separadora de los límites. Por tanto asistimos a una doble preocupación en una región fronteriza, por un lado a la atención por los flujos y por otra a los procesos de cierre o restricción. El barrio boliviano es la expresión de los procesos de interacción que las personas, especialmente bolivianos, tejen en sus constantes idas y venidas más allá de la demanda o los conflictos diplomáticos. Así el análisis multi escalar permite atender a las territorialidades que configuran las movilidades y las prácticas que despliegan las personas en los constantes cruces y cómo éstas transforman un espacio con un marcado sentido de frontera. La dinámica que generan las prácticas sociales fronterizas pone en entredicho el carácter separador del límite porque producen continuidades que son expresión de estrategias de reproducción social que despliegan las personas por motivos laborales o comerciales.

Desde el punto de vista del lugar que ocupa el barrio boliviano en la ciudad como barrio migrante, enclave étnico o territorio circulatorio podemos afirmar que las dos primeras nociones son menos adecuados para definir dicho espacio urbano. La sedentarización y el establecimiento junto a las redes de parentesco o paisanaje son las claves para comprender los barrios migrantes y los enclaves étnicos. La noción de territorio circulatorio y el lugar que ocupa el barrio poseen

${ }_{60}$ TAPIA, Marcela. Frontera y migración en el Norte de Chile a partir del análisis de los censos de población. S. XIX y XXI. 
mayor capacidad explicativa para el caso revisado. Los estudios de Tarrius sobre barrios en distintos puntos de Francia muestran el carácter de dispositivo comercial internacional de barrios como Belsunce en Marsella ${ }^{61}$. A una escala menor, de carácter más bien fronterizo y transfronterizo, el paradigma de la movilidad y el territorio circulatorio permiten dar cuenta de la construcción social de las ciudades y localidades a partir de la complejidad de las relaciones entre los migrantes, los comerciantes y los espacios y su configuración como sujetos nómadas antes que sedentarios $^{62}$. Dicho paradigma permite converger las propuestas descritas anteriormente a través del concepto de territorio circulatorio. Así, la economía migrante -comercial y laboral- tiene una expresión en una espacialidad urbana que en este caso da lugar a un barrio. Además incluye a comerciantes extranjeros, empresarios migrantes, migrantes en sentido tradicional y migrantes móviles, la capacidad para producir y reproducir la interacción y la proximidad. El barrio es una parte del territorio circulatorio en tanto es parte de la producción de un espacio transnacional o transfronterizo donde se conjugan movilidades, un saber circular y una serie de prácticas que vinculan las estructuras económicas y políticas de los países a los que pertenecen, incluso con bastante independencia de los litigios binacionales. Éstas tienen una expresión en el territorio, en este caso circulatorio, donde la transfrontericidad, es decir, las conexiones e interacciones hacia uno y otro lado de la frontera son su rasgo más notorio.

\section{Bibliografía}

ALEGRÍA, Tito. La ciudad y los procesos transfronterizos entre México y Estados Unidos. Frontera Norte, v. 1 n. 2, 1989, p. 53-90.

ALEGRÍA, Tito. Juntos pero no revueltos: ciudades en la frontera México-Estados Unidos. Revista Mexicana de Sociología, v. 62, 2000, p. 89-107.

BABY-COLLIN, Virginia et alii. Mujer, movilidad y territorialización. Análisis cruzado de las migraciones internacionales en México y Bolivia. In GODARD, Henri; SANDOVAL, Godofrefo (eds.). Migración transnacional de los Andes a Europa y Estados Unidos. La Paz: IFEA/PIEB/IRD, 2008, p. 135-166.

BENEDETTI, Alejandro. Lugares de frontera y movilidades comerciales en el sur sudamericano. Una aproximación multiescalar. In DA COSTA, Edgar et alii. Lugares de frontera y movilidades comerciales en el sur sudamericano. Una aproximación multiescalar. Campo Grande: Editora da UFMS, 2011, p. 33-56.

BONACICH, Edna; MODELL, John. The Economic Basis of Ethic Solidarity in the Japanese American Community. Berkely: University of California Press, 1980.

BUCKLEY, Monica. "Amos del universo" y "aves de paso" en la conformación del centro urbano. Lecturas geográficas, v. 2. Madrid: Editorial Complutense, 1999, p. 1045-1053.

CASTILlO, Manuel; NÁJERA, Jéssica. México como país de origen, tránsito y

\footnotetext{
${ }_{61}$ TARRIUS, Pobres en migración..., op. cit.

62 TARRIUS, Alain. Leer, describir, interpretar las circulaciones migratorias: conveniencia de la noción de 'territorio circulatorio'. Los nuevos hábitos de la identidad.
} 
destino de migrantes, una revisión a partir de la EMIF NORTE y la EMIF SUR. In CONSEJO NACIONAL DE POBLACIÓN. 20 años de la Encuesta sobre migración en la frontera norte de México. México, DF, 2014, p. 17-36.

DI MEO, Guy. Géographie tranquille du quotidien. Une analyse de la contribution des sciences sociales et de la géographie à l'étude des pratiques spatiales. Cahiers de Géographie du Québec, v. 43, 1999, p. 75-93.

GARCÉS, Alejandro. Migración peruana en Santiago: prácticas, espacios y economías. Santiago de Chile: RIL Editores, 2015.

GIELIS, Ruben. Borders make the difference: Migrant transnacionalism as a border experience. Tijdschrift voor economische en sociale geografie, v. 100, 2009, p. 598-609.

HEYMAN, Josiah. Construcción y uso de tipologías: movilidad geográfica en la frontera México-Estados Unidos. In ARIZA, Marina; VELASCO, Laura (coords.). Construcción y uso de tipologías: movilidad geográfica en la frontera MéxicoEstados Unidos. México D.F: Instituto de Investigaciones Sociales-UNAM/El Colegio de la Frontera Norte, 2012, p. 419-454.

LABBÉ, Gricel. Del gueto al hipergueto en el centro y pericentro de la ciudad de Iquique. Tesis para optar al título de Geógrafa, Dpto de Geografía, Santiago de Chile: Universidad de Chile, 2014.

LIGHT, Ivan. Ethnic Enternrise in America. Berkeley and Los Angeles: University of California, 1992.

LLANQUE, Ricardo; VILLCA, Edgar. Qamiris aymaras. Desplazamiento e inclusión de elites andinas en la ciudad de Oruro. La Paz: PIEB, 2011.

MATUS, Javiera. Inmigrantes en Chile: más de dos tercios trabajan y el $42 \%$ cotiza en Fonasa. 21.09.2014. Disponible en: <http://www.latercera.com/noticia/ nacional/2014/09/680-596709-9-inmigrantes-en-chile-mas-de-dos-terciostrabajan-y-el-42-cotiza-en-fonasa.shtml>. Consultado: 12.10.2015.

MORALES, Abelardo. Desentrañando fronteras y sus movimientos transnacionales entre pequeños estados. Una aproximación desde la frontera Nicaragua-Costa Rica. In ANGUIANO, María Eugenia; LÓPEZ, Ana María (eds.). Desentrañando fronteras y sus movimientos transnacionales entre pequeños estados. Una aproximación desde la frontera Nicaragua-Costa Rica. Barcelona: Icaria, 2010, p. 185-224.

NEWMAN, David. On borders and power: A theoretical framework. Journal of Borderlands Studies, v. 18, n. 1, 2003, p. 13-25.

OVANDO, Cristian. La noción de soberanía en las propuestas bolivianas para la salida al mar: el caso de Arica trinacional. Diálogo Andino, v. 48, 2015, p. 127-138.

PARELLA, Sonia. El trasvase de desigualdades de clase y etnia entre mujeres: los servicios de proximidad. Papers, v. 60, 2000, p. 275-289.

PARELLA, Sonia. Segregación laboral y 'vulnerabilidad social' de las mujeres inmigrantes a partir de la interacción entre clase social, género y etnia. In SOLÊ, Carlota; FLAQUER, Lluís. El uso de las políticas sociales por las mujeres inmigrantes. Madrid: Instituto de la Mujer, 2005, p. 97-117.

PARELLA, Sonia. Una exploración de las prácticas transfronterizas en la zona urbana Caléxico (Estados Unidos)-Mexicali (México). In TAPIA, Marcela; GONZÁLEZ, Adriana (eds.). Una exploración de las prácticas transfronterizas en la zona urbana Caléxico (Estados Unidos)-Mexicali (México). Santiago de Chile: RIL Editores, 2014, p. 41-69. 
PEREIRA, A.; URIBE, A. Migración comercial boliviana. Acercamiento exploratorio y descriptivo sobre el fenómeno de la migración comercial boliviana y su vinculación con la Zona Franca de Iquique, en la comuna de Iquique. Iquique: Universidad Arturo Prat, 2001.

PORTES, Alejandro. La Sociología en el hemisferio. Hacia una nueva agenda conceptual. Nueva Sociedad, v. 178, 2002, p. 126-144.

PORTES, Alejandro; JENSEN, Leif. The Enclave and the Entrants: Patterns of Ethnic Enterprise in Miami Before and After Mariel. American Sociological Review, v. 54, 1989, p. 929-949.

PORTES, Alejandro; SHAFER, Steven. Revisitando la hipótesis del enclave: Miami veinticinco años después. In PORTES, Alejandro. Revisitando la hipótesis del enclave: Miami veinticinco años después. Barcelona: Anthropos, 2012, p. 117-142.

RIESCO, Alberto. Enclaves y economías étnicos desde la perspectiva de las relaciones salariales. Cuadernos de relaciones laborales, v. 21, 2003, p. 103-125.

SASSONE, Susana; MERA, Carolina. Barrios de migrantes en Buenos Aires: Identidad, cultura y cohesión socioterritorial. 2007. Disponible en: < http:// www.reseau-amerique-latine.fr/ceisal-bruxelles/MS-MIG/MS-MIG-1-Sassone Mera.pdf>. Consultado: 20.10.2015.

SOLÉ, Carlota; PARELLA, Sonia. La inserción de los inmigrantes en el mercado de trabajo. El caso español. In SOLÉ, Carlota (ed.). El impacto de la inmigración en la economía y la sociedad receptora. Barcelona: Anthropos, 2001, p. 11-51.

SOLIMANO, Andrés; TOKMAN, Víctor. Migraciones internacionales en un contexto de crecimiento económico: el caso de Chile. In SOLIMANO, Andrés. (ed.). Migraciones internacionales en América Latina. Booms, crisis y desarrollo. Santiago de Chile: FCE, 2008, p. 185-266.

TAPIA, Marcela. Frontera y migración en el Norte de Chile a partir del análisis de los censos de población. S. XIX y XXI. Revista Geografía Norte Grande, v. 52, 2012, p. 177-198.

TAPIA, Marcela. Migración y movilidad de los trabajadores fronterizos en Tarapacá durante el ciclo del nitrato. 1880-1930. In GONZÁLEZ, Sergio. Migración y movilidad de los trabajadores fronterizos en Tarapacá durante el ciclo del nitrato. 1880-1930. Santiago de Chile: RIL, 2013, p. 163-194.

TAPIA, Marcela. Extranjeros fronterizos en las regiones extremas de Chile: entre migración y circulación. 1990-2014. In ROJAS, Nicolás; VICUÑA, José Tomás (eds.). Extranjeros fronterizos en las regiones extremas de Chile: entre migración y circulación. 1990-2014. Santiago: Ciudadano Global/OIM, 2014, p. 31-55.

TAPIA, Marcela. Frontera, movilidad y circulación reciente de peruanos y bolivianos en el norte de Chile Estudios atacameños. Arqueología y Antropología Surandinas, v. 50, 2015, p. 195-213.

TAPIA, Marcela; GONZÁLEZ, Adriana. Regiones fronterizas, migración y los desafíos para los Estados nacionales latinoamericanos. Santiago de Chile: RIL Editores, 2014. TAPIA, Marcela; PARELLA, Sonia. Las regiones fronterizas para el estudio de la migración y la circulación. Un análisis de dos casos ilustrativos. In GUIZARDI, Menara. Las regiones fronterizas para el estudio de la migración y la circulación. Un análisis de dos casos ilustrativos. Ocho Libros, 2015, p. 173-206.

TAPIA, Marcela; RAMOS, Romina. Mujeres migrantes fronterizas en Tarapacá a 
principios del siglo XXI. El cruce de las fronteras y las redes de apoyo. Polis. Revista Latinoamericana, v. 12, n. 35, 2013, p. 229-257.

TARRIUS, Alain. Leer, describir, interpretar las circulaciones migratorias: conveniencia de la noción de 'territorio circulatorio'. Los nuevos hábitos de la identidad. Relaciones, v. 21, n. 83, 2000, p. 39-66.

TARRIUS, Alain. Migrantes pobres y globalización de las economías: el transnacionalismo migratorio en Europa meridional. In LARA, Sara María. Migraciones de trabajo y movilidad territorial. México D.F: Miguel Ángel Porrúa, 2010, p. 101-122.

TARRIUS, Alain. Pobres en migración, globalización de las economías y debilitamiento de los modelos integradores: el transnacionalismo migratorio en Europa meridional. Empiria. Revista de metodología de Ciencias Sociales, v. 19, 2010, p. 133-156.

TASSI, Nico et alii. El desborde económico popular en Bolivia. Comerciantes aymaras en el mundo global. Nueva Sociedad, v. 214, 2012, p. 93-105.

TASSI, Nico et alii. "Hacer plata sin plata". El desborde de los comerciantes populares en Bolivia. La Paz: PIEB, 2013.

ZAPATA-BARRERO, Ricard; FERRER-GALLARDO, Xavier. Las fronteras en la época de la movilidad. In IDEM (coords.). Fronteras en movimiento: migraciones hacia la Unión Europea en el contexto Mediterráneo. Barcelona: Edicions Bellaterra, 2012, 11-56.

ANEXOS

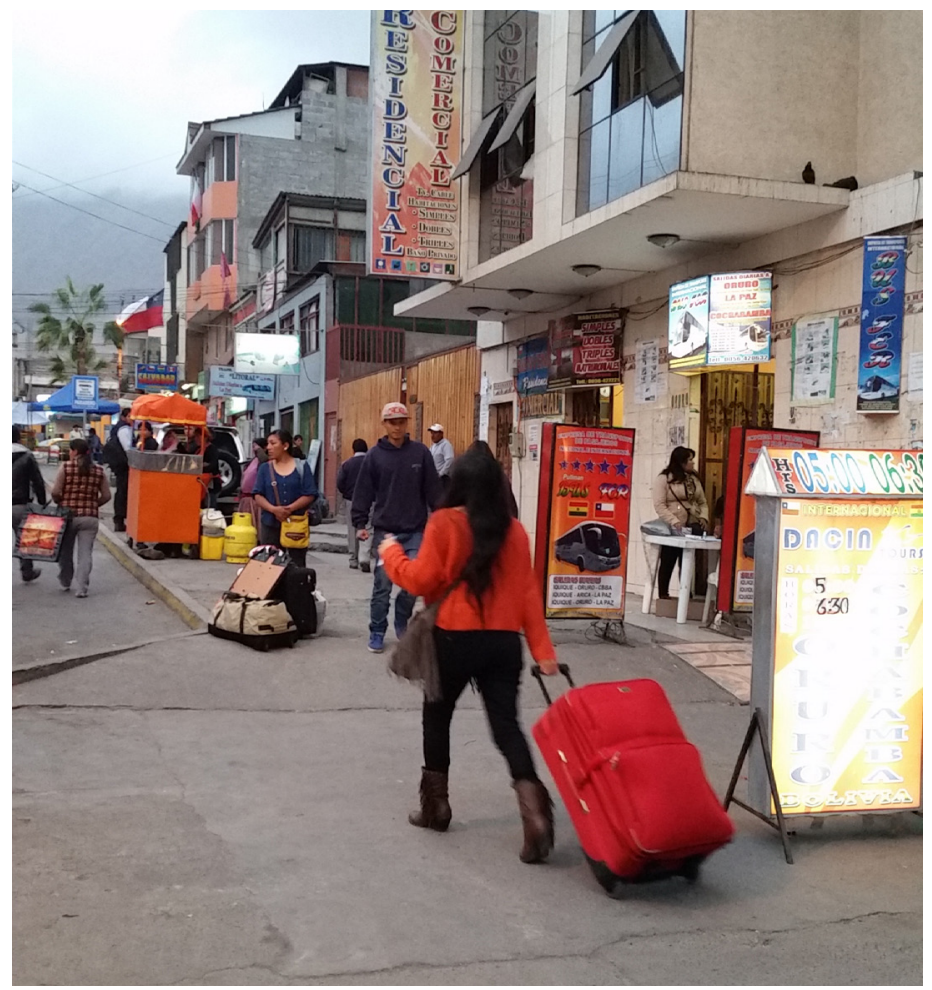



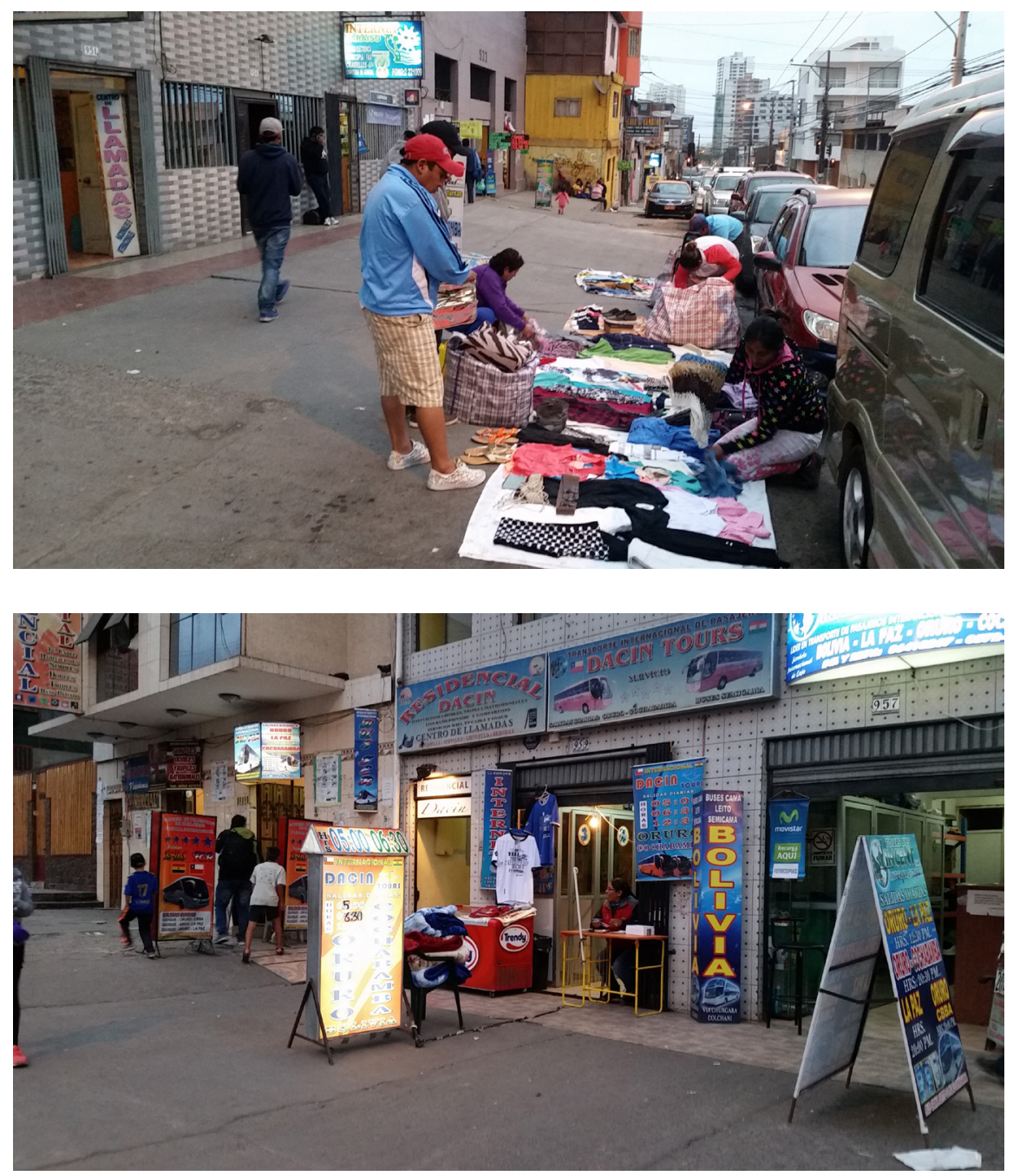

Recibido para publicación en 23.12.2015

Aceptado para publicación en 10.03.2016 Received for publication in December 23rd, 2015

Accepted for publication in March 10 ${ }^{\text {th }}, 2016$

ISSN impresso 1980-8585

ISSN eletrônico 2237-9843

http://dx.doi.org/10.1590/1980-85852503880004709 